\title{
COEFFICIENT ESTIMATE FOR A SUBCLASS OF CLOSE-TO-CONVEX FUNCTIONS WITH RESPECT TO SYMMETRIC POINTS
}

\author{
B. S. MEHROK, GAGANDEEP SINGH AND DEEPAK GUPTA
}

\begin{abstract}
For reals $A, B, C, D$ such that $-1 \leq D \leq B<A \leq C \leq 1$, a subclass $K_{s}(A, B ; C, D)$ of analytic functions $f(z)=z+\sum_{k=2}^{\infty} a_{k} z^{k}$ in the open unit disc $E=\{z:|z|<1\}$ is introduced. The object of the present paper is to determine the coefficient estimate for functions $f(z)$ belonging to the class $K_{s}(A, B ; C, D)$.
\end{abstract}

\section{Introduction}

Let $U$ denote the class of functions

$$
w(z)=\sum_{k=1}^{\infty} c_{k} z^{k}
$$

which are regular in the unit disc $E=\{z:|z|<1\}$ and satisfying the conditions

$$
w(0)=0 \text { and }|w(z)|<1, \quad z \in E .
$$

Let $S$ be the class of functions

$$
f(z)=z+\sum_{k=2}^{\infty} a_{k} z^{k}
$$

which are regular and univalent in $E$.

Let $S_{s}^{*}$ denote the class of functions $f(z) \in S$ and satisfying the condition

$$
\operatorname{Re}\left(\frac{z f^{\prime}(z)}{f(z)-f(-z)}\right)>0, \quad z \in E .
$$

These functions are called Starlike with respect to symmetric points and were introduced by Sakaguchi [4]. After this Goel and Mehrok [2] introduced by a sub-class $S_{s}^{*}(A, B)$ of $S_{s}^{*}$. $S_{s}^{*}(A, B)$ be the class of functions $f(z) \in S$ which satisfy the condition

$$
\frac{2 z f^{\prime}(z)}{f(z)-f(-z)}<\frac{1+A z}{1+B z}, \quad-1 \leq B<A \leq 1, z \in E .
$$

Corresponding author: Gagandeep Singh.

2000 Mathematics Subject Classification. 30C45.

Key words and phrases. Starlike with respect to symmetric points, close-to convex with respect to symmetric points, coefficient estimate. 
Let $K_{S}(A, B ; C, D)$ be the class consisting of functions $f(z) \in S$ and satisfying the condition

$$
\frac{2 z f^{\prime}(z)}{(g(z)-g(-z))}<\frac{1+C z}{1+D z}, \quad-1 \leq D \leq B<A \leq C \leq 1, z \in E
$$

where

$$
g(z)=z+\sum_{k=2}^{\infty} b_{k} z^{k} \in S_{s}^{*}(A, B) .
$$

Obviously $K_{s} \equiv K_{S}(1,-1 ; 1,-1)$ and $K_{S}(A, B)=K_{S}(A, B ; 1,-1)$.

By definition of subordination it follows that $f \in K_{s}(A, B ; C, D)$ if and only if

$$
\frac{2 z f^{\prime}(z)}{g(z)-g(-z)}=\frac{1+C w(z)}{1+D w(z)}=P(z), \quad w(z) \in U
$$

where

$$
P(z)=1+\sum_{k=1}^{\infty} p_{k} z^{k}
$$

We obtain the coefficient estimate for the class $K_{S}(A, B ; C, D)$.

\section{Some preliminary lemmas}

We shall require the following lemmas.

Lemma 2.1. If $P(z)$ is given by (1.3), then

$$
\left|p_{n}\right| \leq(C-D)
$$

This lemma is due to Goel and Mahrok [2].

Lemma 2.2. Let $g(z)=z+\sum_{k=2}^{\infty} b_{k} z^{k} \in S_{s}^{*}(A, B)$, then for $n \geq 1$,

$$
\left|b_{2 n}\right| \leq \frac{(A-B)}{n ! 2^{n}} \prod_{j=1}^{n-1}(A-B+2 j)
$$

and

$$
\left|b_{2 n+1}\right| \leq \frac{(A-B)}{n ! 2^{n}} \prod_{j=1}^{n-1}(A-B+2 j) .
$$

This result was established by Goel and Mahrok [2]. 


\section{Main result}

Theorem 3.1. Let $f \in K_{s}(A, B ; C, D)$, then for $n \geq 1$,

$$
\begin{aligned}
\left|a_{2 n}\right| & \leq \frac{(C-D)}{n ! 2^{n}} \prod_{j=1}^{n-1}(A-B+2 j) \\
\left|a_{2 n+1}\right| & \leq \frac{1}{2 n+1}\left\{\left[(C-D)+\frac{(A-B)}{2 n}\right]\left[\frac{1}{(n-1) ! 2^{n-1}} \prod_{j=1}^{n-1}(A-B+2 j)\right]\right\} .
\end{aligned}
$$

Proof. As $g \in S_{s}^{*}(A, B)$, it follows that

$$
\begin{aligned}
2 z g^{\prime}(z) & =(g(z)-g(-z)) K(z) \quad \text { for } z \in E \\
\text { where } \quad K(z) & =1+d_{1} z+d_{2} z^{2}+d_{3} z^{3}+\cdots .
\end{aligned}
$$

On equating the coefficients of like powers of $z$ in (3.3), we get

$$
\begin{aligned}
& 2 b_{2}=d_{1}, \quad 2 b_{3}=d_{2}, \\
& 4 b_{4}=d_{3}+b_{3} d_{1}, \quad 4 b_{5}=d_{4}+b_{3} d_{2},
\end{aligned}
$$

Continuing in this way, we have

$$
\begin{aligned}
& 2 n b_{2 n}=d_{2 n-1}+b_{3} d_{2 n-3}+b_{5} d_{2 n-5}+\cdots+b_{2 n-1} d_{1} \\
& 2 n b_{2 n+1}=d_{2 n}+b_{3} d_{2 n-2}+b_{5} d_{2 n-4}+\cdots+b_{2 n-1} d_{2}
\end{aligned}
$$

From (1.3) and (1.4), we have

$$
\begin{aligned}
z+2 & a_{2} z^{2}+3 a_{3} z^{3}+\cdots+2 n a_{2 n} z^{2 n}+(2 n+1) a_{2 n+1} z^{2 n+1}+\cdots \\
= & \left(z+b_{3} z^{3}+b_{5} z^{5}+\cdots+b_{2 n-1} z^{2 n-1}+b_{2 n+1} z^{2 n+1}+\cdots\right) \\
& \cdot\left(1+p_{1} z+p_{2} z^{2}+\cdots+p_{2 n} z^{2 n}+p_{2 n+1} z^{2 n+1}+\cdots\right) .
\end{aligned}
$$

On equating the coefficients, we otain

$$
\begin{aligned}
& 2 a_{2}=p_{1}, \quad 3 a_{3}=p_{2}+b_{3}, \\
& 4 a_{4}=p_{3}+b_{3} p_{1}, \quad 5 a_{5}=p_{4}+b_{3} p_{2}+b_{5},
\end{aligned}
$$

and so on

$$
\begin{aligned}
& 2 n a_{2 n}=p_{2 n-1}+b_{3} p_{2 n-3}+b_{5} p_{2 n-5}+\cdots+b_{2 n-1} p_{1}, \\
& (2 n+1) a_{2 n+1}=p_{2 n}+b_{3} p_{2 n-2}+b_{5} p_{2 n-4}+\cdots+b_{2 n-1} p_{2}+b_{2 n+1} .
\end{aligned}
$$

Using Lemma 2.1 and equation (3.8), we get

$$
2\left|a_{2}\right| \leq C-D, \quad 3\left|a_{3}\right| \leq(C-D)+\frac{(A-B)}{2} .
$$


Again applying Lemma 2.1 and using equation (3.4) and (3.5), we obtain from (3.9)

$$
4\left|a_{4}\right| \leq \frac{(C-D)(A-B+2)}{2}, \quad 5\left|a_{5}\right| \leq \frac{(A-B+2)[(A-B)+4(C-D)]}{8} .
$$

It follow that (3.1) and (3.2) hold for $n=1,2$.

We now prove (3.1) and (3.2) by induction.

(3.10) and (3.11) in conjunction with Lemma 2.1 yield

$$
\left|a_{2 n}\right| \leq \frac{(C-D)}{2 n}\left[1+\sum_{k=1}^{n-1}\left|b_{2 k+1}\right|\right] .
$$

and

$$
\left|a_{2 n+1}\right| \leq \frac{1}{2 n+1}\left\{(C-D)\left[1+\sum_{k=1}^{n-1}\left|b_{2 k+1}\right|\right]+\left|b_{2 n+1}\right|\right\} .
$$

Again by using Lemma 2.1 in (3.7), we have

$$
\left|b_{2 n+1}\right| \leq \frac{(A-B)}{2 n}\left[1+\sum_{k=1}^{n-1}\left|b_{2 k+1}\right|\right] .
$$

From (3.13) and (3.14), we obtain

$$
\left|a_{2 n+1}\right| \leq \frac{1}{2 n+1}\left\{\left[(C-D)+\frac{(A-B)}{2 n}\right]\left[1+\sum_{k=1}^{n-1}\left|b_{2 k+1}\right|\right]\right\} .
$$

We assume that (3.1) and (3.2) holds for $k=3,4, \ldots,(n-1)$.

Using Lemma 2.2 in (3.12) and (3.15), we obtain

$$
\left|a_{2 n}\right| \leq \frac{(C-D)}{2 n}\left[1+\sum_{k=1}^{n-1} \frac{(A-B)}{k ! 2^{k}} \prod_{j=1}^{k-1}(A-B+2 j)\right]
$$

and

$$
\begin{aligned}
\left|a_{2 n+1}\right| \leq & \frac{1}{2 n+1}\left\{(C-D)\left[1+\sum_{k=1}^{n-1} \frac{(A-B)}{k ! 2^{k}} \prod_{j=1}^{k-1}(A-B+2 j)\right]\right. \\
& \left.+\frac{(A-B)}{2 n}\left[1+\sum_{k=1}^{n-1} \frac{(A-B)}{k ! 2^{k}} \prod_{j=1}^{k-1}(A-B+2 j)\right]\right\} .
\end{aligned}
$$

In order to prove (3.1), it is sufficient to show that

$$
\frac{(C-D)}{2 m}\left[1+\sum_{k=1}^{m-1} \frac{(A-B)}{k ! 2^{k}} \prod_{j=1}^{k-1}(A-B+2 j)\right]=\frac{(C-D)}{m ! 2^{m}} \prod_{j=1}^{m-1}(A-B+2 j), \quad(m=3,4, \ldots)
$$

(3.18) is valid for $m=3$.

Let us suppose that (3.18) is true for all $m, 3<m \leq(n-1)$. Then from (3.16), we have

$$
\frac{(C-D)}{2 n}\left[1+\sum_{k=1}^{n-1} \frac{(A-B)}{k ! 2^{k}} \prod_{j=1}^{k-1}(A-B+2 j)\right]
$$




$$
\begin{aligned}
= & \frac{(n-1)}{n}\left\{\frac{(C-D)}{2(n-1)}\left[1+\sum_{k=1}^{n-2} \frac{(A-B)}{k ! 2^{k}} \prod_{j=1}^{k-1}(A-B+2 j)\right]\right\} \\
& +\frac{(C-D)}{2 n} \cdot \frac{(A-B)}{(n-1) ! 2^{n-1}} \prod_{j=1}^{n-2}(A-B+2 j) \\
= & \frac{(n-1)}{n} \cdot \frac{(C-D)}{(n-1) ! 2^{n-1}} \prod_{j=1}^{n-2}(A-B+2 j) \\
& +\frac{(C-D)}{2 n} \cdot \frac{(A-B)}{(n-1) ! 2^{n-1}} \prod_{j=1}^{n-2}(A-B+2 j) \\
= & \frac{(C-D)}{(n-1) ! 2^{n-1}} \prod_{j=1}^{n-2}(A-B+2 j) \frac{(A-B+2(n-1))}{2 n} \\
= & \frac{(C-D)}{n ! 2^{n}} \prod_{j=1}^{n-1}(A-B+2 j) .
\end{aligned}
$$

Thus (3.18) holds for $m=n$ and hence (3.1) follows.

Now from (3.17), we have

$$
\left|a_{2 n+1}\right| \leq \frac{1}{2 n+1}\left\{\left[(C-D)+\frac{(A-B)}{2 n}\right]\left[1+\sum_{k=1}^{n-1} \frac{(A-B)}{k ! 2^{k}} \prod_{j=1}^{k-1}(A-B+2 j)\right]\right\} .
$$

From (3.18) , we have

$$
1+\sum_{k=1}^{n-1} \frac{(A-B)}{k ! 2^{k}} \prod_{j=1}^{k-1}(A-B+2 j)=\frac{1}{(n-1) ! 2^{n-1}} \prod_{j=1}^{n-1}(A-B+2 j)
$$

From (3.19) and (3.20), we have

$$
\left|a_{2 n+1}\right| \leq \frac{1}{2 n+1}\left\{\left[(C-D)+\frac{(A-B)}{2 n}\right]\left[\frac{1}{(n-1) ! 2^{n-1}} \prod_{j=1}^{n-1}(A-B+2 j)\right]\right\}
$$

which proves (3.2).

Putting $A=C=1$ and $B=D=-1$ in the above result, we get the following

Corollary 1. Let $f(z)$ be schlicht and starlike with respect to symmetric points in the unit disc $E$, having the form $f(z)=z+\sum_{k=2}^{\infty} a_{k} z^{k}$, then

$$
\left|a_{n}\right| \leq 1 \text { for any natural number } n \text {. }
$$

This result was proved by Das and Singh [1].

For $C=1$ and $D=-1$, we have the following result for the class $K_{s}(A, B)$. 
Corollary 2. Let $f \in K_{s}(A, B)$, then for $n \geq 1$,

$$
\left|a_{2 n+1}\right| \leq \frac{1}{2 n+1}\left\{\left[2+\frac{(A-B)}{2 n}\right]\left[\frac{1}{(n-1) ! 2^{n-1}} \prod_{j=1}^{n-1}(A-B+2 j)\right]\right\} .
$$

Remark. Janteng and Halim [3] proved that, for $f \in K_{S}(A, B)$ and for $n \geq 1$,

$$
\left|a_{2 n+1}\right| \leq \frac{(A-B)}{(2 n+1)(n-1) ! 2^{n-1}} \prod_{j=1}^{n-1}(A-B+2 j) .
$$

This result is not justified.

\section{References}

[1] R. N. Das and P. Singh, On subclasses of schlicht mapping, Ind. J. Pure Appl. Math., 8(1977), 864-872.

[2] R. M. Goel and Beant Singh Mehrok, A subclass of starlike functions with respect to symmetric points, Tamkang J. math., 13(1982), 11-24.

[3] Aini Janteng and Suzeini Halim, Coefficient estimate for a subclass of close-to-convex functions with respect to symmetric points, Int. Journal of Math. Analysis, 3(2009), 309-313.

[4] K. Sakaguchi, On a certain univalent mapping, J. Math. Soc. Japan, 11(1959), 72-80.

\#643 E, B.R.S. Nagar, Ludhiana - 141001, Punjab, India.

Department of Mathematics, Miri Piri Khalsa College, Bhadaur (Barnala)-148102, Punjab(India).

E-mail: kamboj.gagandeep@yahoo.in

Department of Mathematics, M. M. University, Mullana-Ambala, Haryana, India. 